\title{
L’analyse De La Place De La Valeur Perçue Comme Un Déterminant Stratégique Des Enseignes De La Grande Distribution Marocaine : Cas De La Région Souss Massa
}

\author{
Bouchouar Otman, (Doctorant) \\ Akhlaffou Mohamed, (Doctorant) \\ Souaf Malika, (Professeure Chercheure) \\ El Wazani Youssef, (Professeur Chercheur)
}

Equipe de Recherche en Management et Performance des Organisations

Publiques, Privées et de l’Economie Sociale, Ecole Nationale de Commerce et de Gestion, Université Ibn Zohr, Agadir, Maroc

\section{doi: 10.19044/esj.2016.v13n2p141 URL:http://dx.doi.org/10.19044/esj.2016.v13n2p141}

\begin{abstract}
This article tries to highlight the effects of the perceived value on satisfaction, and determines, among the components of the value, the major sources of shopping behavior. This leads us to a reflection in order to promote in one hand, the concept of perceived value among the mass-market retailing and on the other hand, to define the reasons for attendance, stimulation and consumer's escape inside the point of sale.

For this purpose, our exploratory research highlights the typology of value of Holbrook (1994, 1999). Relying on series of interviews with customers of large retail in the city of Agadir, the point of sale is no longer considered as a simple place of purchase. It shows different types of value, such as utilitarian values, hedonist values and social values. Our results are able to validate this idea and therefore tend to position the point of sale as a key place for the formulation of marketing strategies of retail chains and thus to be seen as a creator of value.
\end{abstract}

Keywords: Perceived Value, Consumer satisfaction, Frequentation, Shopping behavior, Retailing

\section{Résumé}

Cet article cherche à mettre en évidence les effets de la valeur perçue sur la satisfaction des clients, et de déterminer, parmi les composantes de la valeur, les sources majeures du comportement de magasinage. Ceci nous 
conduit à une réflexion dans le but de valoriser d'une part, le concept de la valeur perçue chez les entreprises de la grande distribution, et d'autre part, de définir les motifs de fréquentation, de stimulation et d'évasion des consommateurs à l'intérieur du point de vente.

À cet effet, notre recherche exploratoire met en exergue la typologie de la valeur d’Holbrook (1994, 1999). En nous appuyant sur une série d'entretiens avec des clients des grandes enseignes de distribution de la ville d'Agadir, le point de vente n'est plus considéré comme un simple lieu d'achat. Il en ressort différents types de valeurs, telles que les valeurs utilitaires, les valeurs hédonistes et les valeurs sociales. Nos résultats sont en mesure de valider cette idée et tendent dès lors à positionner le lieu de vente comme un endroit clé de formulation des stratégies marketing des enseignes de distributions et donc être vu comme créateur de valeur.

Mots-Clés : Valeur perçue, Satisfaction du consommateur, Fréquentation de point de vente, Comportement de magasinage, enseignes de la grande distribution

\section{Introduction}

Depuis quelques années, plusieurs recherches se sont attachées à mieux comprendre la valeur perçue des consommateurs, notamment dans le domaine de la distribution. Toutefois, Le concept de valeur perçue fait l'objet de plusieurs définitions en marketing. Sa conceptualisation et sa mesure sont encore confuses et posent aujourd'hui un certain nombre d'interrogations.

En effet, les professionnels du marketing cherchent à déployer leurs efforts afin de mieux appréhender les aspects et les spécificités de ce construit ainsi que sa contribution à la satisfaction client. Cet article cherche donc à contribuer à l'amélioration de la compréhension de la valeur perçue de magasinage en intégrant les trois dimensions identifiées par Holbrook, dans le contexte Marocain.

Ceci nous conduit à une réflexion dans le but de valoriser d'une part, le concept de la valeur perçue chez les entreprises de la grande distribution et d'autre part, de définir les motifs de plaisir, de stimulation et d'évasion chez les consommateurs à l'intérieur du point de vente. A cet effet, il sera intéressant de présenter et de spécifier les fondements conceptuels de la valeur perçue, ainsi que les motivations de fréquentation des lieux de vente, avant d'étudier les relations entre ces construits théoriques et leurs effets sur la satisfaction des clients. Par la suite, un modèle d'analyse, sera conçu théoriquement et testé empiriquement, dans le but d'examiner de près les différents liens entre les composantes de la valeur perçue. 
L’article sera dédiée à la mise au point d’une étude qualitative qui permettra d'en identifier les différentes dimensions posées par Holbrook, en nous appuyant sur une série d'entretiens semi-directifs avec des clients des grandes enseignes de distribution de la ville d'Agadir. Puis nous présenterons les résultats des analyses exploratoires obtenus, dans la perspective de répondre à la question suivante : Quels sont les déterminants de la valeur perçue du consommateur marocain lors de sa fréquentation des points de vente. Ce qui nous permettra de déterminer les principaux apports, les limites et les voies de développement utiles pour une gestion commerciale performante de la grande distribution.

\section{Revue de la littérature : cadre conceptuel de la valeur perçue en marketing}

Aujourd'hui, la valeur perçue est devenue l'une des exigences les plus importantes dans le domaine de la grande distribution. Ce concept constitue la source de motivation du consommateur envers les grandes enseignes, et elle donne un sens et oriente leurs actes. Par ailleurs, dans cette partie de l'article, nous nous intéressons à la définition de la valeur perçue du magasin, plus particulièrement celle proposée par Holbrook (1999) et les différentes approches et composantes de la valeur perçue en marketing.

\section{Définition et composantes clés de la valeur perçue en marketing}

La notion de valeur est largement utilisée en sciences de gestion (Bréchet et Desreumaux, 1998). C'est pour cette raison que plusieurs travaux ont fait de la valeur leur centre d’intérêt. La valeur représente un paradigme important tant pour les études du comportement d'achat du consommateur (Zeithaml, 1988 ; Desmet et Zollinger, 1997 ; Day, 2002), qu’en matière de marketing relationnel (Anderson et Narus (1995), cités par Kaabachi, 2007). Il est néanmoins important de signaler que la variabilité sémantique de ce terme permet de le considérer sous différents angles. Cela prête à diverses interprétations (Rivière, 2007).

Dans ce contexte, il est nécessaire de définir et comprendre ce que représente la valeur d'un objet par le client, dans la mesure où, comme le soulignent Aurier et al. (1998), «l'acquisition d'un avantage compétitif soutenable présuppose la création et la distribution régulière d'une valeur supérieure aux clients ». Dans la littérature marketing, la notion de valeur est considérée comme un concept essentiel attaché à la notion d’échange en marketing (Holbrook, 1994). Selon Ulaga et Eggert (2002) et Marteaux (2007), la valeur perçue d'un produit ou d'un service, par un client, est généralement considérée comme le résultat d'un écart entre les coûts et les bénéfices, s’inscrivant dans une perspective d'échange. 
Traditionnellement, la valeur perçue est définie comme étant le résultat de la confrontation entre les bénéfices perçus et les coûts perçus (Aurier, Evrard et N’Goala, 2004), ou encore comme l'évaluation globale du consommateur fondée sur l'utilité d'un produit, basée sur les perceptions de ce qui est reçu et ce qui est donné (Zeithaml, 1988). Ainsi, le consommateur percevra une valeur perçue positive si les bénéfices sont supérieurs au prix et vice-versa (Woodall, 2003).

De nombreux auteurs ont souvent démontré le rôle essentiel de la valeur perçue dans le comportement du consommateur. Ils considèrent que la valeur perçue doit être placée au centre de la politique marketing, compte tenu de l'importance qu'elle représente pour les entreprises en termes d'avantage concurrentiel (Lai, 1995 ; Woodruff, 1997 ; Aurier et al., 1998, 2000, 2004 ; Huber et Herrmann, 2000 ; Sweeney et Soutar, 2001 ; Khalifa, 2004). D'ailleurs, dans un cadre théorique de la relation du prospect avec le point de vente, la valeur perçue s'inscrite en trois étapes à savoir : le choix du magasin, résultant d'une formation multi-attributs des préférences aboutissant à la construction d'un comportement d'achat (Cliquet, 1992), la fréquentation du magasin, source d'expérience liée au concept de satisfaction (Filser, des Garets et Paché, 2001). Enfin, grâce à la valeur perçue de sa prestation, l'adoption d'un comportement de fidélité à l'enseigne (Evrard et Aurier, 1996).

Plusieurs classifications de la valeur perçue ont été proposées et démontrées dans la littérature en marketing. En particulier, Tauber (1972) affirme que la valeur perçue peut être utilitaire, hédoniste, sociale ou psychologique. En outre, Sweeney et Soutar (2001) considèrent que la valeur perçue est le résumé de quatre dimensions à savoir : La valeur émotive qui est le résultat d'un état sentimental et affectif qu'un produit est susceptible de provoquer. La valeur sociale qui représente la disposition du produit à renforcer sa propre considération sociale. La valeur fonctionnelle ou encore la qualité qui est la performance espérée du produit, et en fin la valeur prix ou monétaire représentée par la réduction des coûts perçus à court terme et à long terme.

Holbrook (1994, 1999), a proposé une typologie de la valeur de consommation. En outre, ses travaux constituent un apport majeur dans le cadre de cette approche. Celle-ci s'articule autour de trois dimensions clés à savoir ; une dimension ontologique (orientation intrinsèque ou extrinsèque), une dimension praxéologique (orientation active ou passive) et une dimension sociale (orientation individuelle ou interpersonnelle). La cohérence de ces trois dimensions conduit Holbrook (1999) à classifier huit facettes de la valeur (voir tableau $\mathrm{n}^{\circ} 1$ ) susceptibles d'être perçues à la suite d'une expérience de consommation. 
Tableau $\mathrm{n}^{\circ} 1$ : La typologie des sources de la valeur d'Holbrook

\begin{tabular}{|c|c|c|c|}
\cline { 2 - 4 } \multicolumn{2}{c|}{} & Extrinsèque & Intrinsèque \\
\hline \multirow{2}{*}{$\begin{array}{c}\text { Orientation vers soi } \\
\text { (individuelle) }\end{array}$} & Actif & Efficience & Ludique \\
\cline { 2 - 4 } & Réactif & Excellence & Esthétique \\
\hline $\begin{array}{c}\text { Orientation vers les } \\
\text { autres (sociale) }\end{array}$ & Actif & Statut & Ethique \\
\cline { 2 - 4 } & Réactif & Estime & Spiritualiste \\
\hline
\end{tabular}

Source : Holbrook M. (1999).

\section{Méthodologie de recherche}

Notre recherche s'inscrit dans une logique de compréhension et d'exploration (Evrard et al., 2003). Bergadaà (1992) affirme que dès lors que la recherche se trouve dans une approche de découverte d'un terrain peu connu dans le cadre de sa discipline, il est tout à fait légitime d'adopter une approche de type inductif. Il s'agit de mieux comprendre dans un contexte marocain, les composantes et les dimensions de la valeur perçue dans la grande distribution et d'explorer la nature ainsi que la forme des motivations, des perceptions et des attentes des consommateurs, et donc à faire comprendre aux entreprises de la grande distribution que la valeur perçue est une variable stratégique pour leur avenir.

Ainsi, étant donné que l'objectif de cette étude est de recourir à un phénomène qui a été auparavant traité dans une approche purement quantitative (Bloch et al., 1983, 1989 ; Jarboe et McDaniel, 1987). Dont les connaissances restent cependant floues et peu étudiées en profondeur selon les chercheurs (Georges et Al, 2003 ; Cova, 2005 ; Ulaga et Egert, 2002 ; Ulaga et $\mathrm{Al}, 2005,2006)$, le recours à une recherche qualitative se justifie par la volonté de redécouvrir ce comportement de fréquentation des points de vente et de revisiter la notion d'image que véhicule l'enseigne, ainsi que sa perception auprès du consommateur. Et, donc, à valoriser la place de la valeur perçue dans la stratégie globale des entreprises de la grande distribution.

\section{Présentation de l'étude qualitative " entretiens clients "}

Notre ambition, à partir d'une étude qualitative réalisée auprès de clients de grandes surfaces au Maroc, est de mettre en place les principales composantes de la valeur perçue à l'issue d'une expérience de magasinage, en nous basant sur la typologie d'Holbrook (1994, 1999). Le constat consiste à comprendre dans quelle mesure la typologie de Holbrook pourrait-elle s'appliquer au domaine de la grande distribution marocaine, et plus précisément celui de la ville d'Agadir, et donc d'identifier les sources et les principaux fondements des différents types de valeur. C'est dans ce cadre que les clients étaient interviewés sur les motifs de fréquentation et de choix de l'enseigne, image de l'enseigne, sur le type de valeurs attendues et l'interaction avec l'enseigne. 
En nous référant à la revue de littérature existante, et aux besoins en informations exigées par la recherche, nous nous sommes appuyé sur un guide d'entretien rédigé en suivant le raisonnement de l'entonnoir, c'est-àdire en partant de notions générales de notre recherche aux questions strictement liées à l'objet de l'étude.

\section{Présentation de l'échantillon}

La sélection des points de vente et des répondants a été effectuée sur la base d'un échantillonnage par convenance. Les points de vente retenus sont les grandes et moyennes surfaces populaires situées dans la ville d'Agadir, qui offrent des produits et services très demandés par le consommateur Marocain : Groupe Marjane, Aswak Assalam et Carrefour.

Notre échantillon présente les caractéristiques suivantes :

Tableau $n^{\circ} 2$ : Profil de l'échantillon de l'enquête qualitative

\begin{tabular}{|c|c|c|c|}
\hline \multicolumn{2}{|c|}{ Profil } & Effectifs & Fréquence \\
\hline \multirow{4}{*}{ Age } & Homme & 25 & $47 \%$ \\
\cline { 2 - 4 } & Femme & 28 & $53 \%$ \\
\cline { 2 - 4 } & Total & 53 & - \\
\hline \multirow{4}{*}{ Sexe } & De 15 à 29 ans & 24 & $45 \%$ \\
\cline { 2 - 4 } & De 30 à 44 ans & 14 & $26 \%$ \\
\cline { 2 - 4 } & De 45 à 59 ans & 4 & $21 \%$ \\
\cline { 2 - 4 } & Plus de 60 ans & 53 & $8 \%$ \\
\hline \multirow{5}{*}{ Profession } & Total & 4 & $100 \%$ \\
\cline { 2 - 4 } & Cadre supérieur & 6 & $7 \%$ \\
\cline { 2 - 4 } & Cadre moyen & 6 & $11 \%$ \\
\cline { 2 - 4 } & Profession libérale & 11 & $11 \%$ \\
\cline { 2 - 4 } & Fonctionnaire & 3 & $6 \%$ \\
\cline { 2 - 4 } & Employé & 3 & $34 \%$ \\
\cline { 2 - 4 } & Retraité & 53 & $18 \%$ \\
\cline { 2 - 4 } & Eans activité professionnelle & & $100 \%$ \\
\cline { 2 - 4 } & Total & & \\
\hline
\end{tabular}

Source : élaboration personnelle.

\section{Déroulement de la collecte de données}

Le recueil de données a porté sur un échantillon de 53 clients des grandes surfaces à travers une étude qualitative basée sur des entretiens semi-directifs, conduite entre le 26 septembre et le 03 octobre 2016, d'une durée moyenne de 20 minutes par entretien. Il s'agit bien d’un échantillon par convenance constitué d'une diversification des profils (tableau $\mathrm{n}^{\circ} 2$ ). $\mathrm{La}$ collecte des informations s'est effectuée à l'intérieur et à la sortie des caisses des enseignes de distribution de la ville d'Agadir. Les entretiens se sont déroulés auprès des clients à l'intérieur du magasin et qui s'apprêtaient à quitter le magasin. 
Sur ce corpus ont été pratiquées trois analyses différentes. Nous avons mis en œuvre des techniques d'analyse de contenu « manuelles». Une analyse de contenu thématique a permis de catégoriser et de dénombrer les thèmes repris par chaque répondant aux éléments suivants :

- Les motifs de fréquentation et choix de magasin : identifier les composantes clés de la valeur perçue par le consommateur lors de sa visite à l'enseigne ;

- $\quad$ Les effets de la valeur perçue de magasinage sur la satisfaction des clients : définir les sources de valeur perçue ;

- $\quad$ Les déterminants de la valeur perçue de magasinage : identifier les composantes clés de la valeur qui influence l'acte d'achat du consommateur.

\section{Résultats et discussion}

\section{Les motifs de fréquentation et choix d'un point de vente}

L'étude s'inscrite, par essence, dans un contexte marocain. Nous traiterons d'abord les motifs de fréquentation et le choix des points de vente. Cette partie de l'analyse visera à identifier les composantes clés de la valeur perçue par le consommateur lors de sa visite à l'enseigne.

L’analyse des données collectées, nous a permis de confirmer la pertinence de la pensé de Holbrook (2009) en termes de typologies de sources de la valeur. Dans la suite, nous exposons les composantes de la valeur (valeur utilitaire, valeur hédonique, valeur sociale) associée aux motivations de visite et de choix du point de vente, sur un tableau récapitulatif, les différentes facettes et l'occurrence attachées à chacune d'entre elles.

\section{La valeur utilitaire}

L'analyse des résultats, sur la base de la typologie de Holbrook (2009), met en exergue quatre facettes de composantes de la valeur utilitaire au sein des grandes surfaces. Nous proposons dans ce qui suit (voir tableau $\mathrm{n}^{\circ} 3$ ) la classification de la valeur utilitaire selon le discours des répondants :

Tableau ${ }^{\circ} 3$ : L’occurrence de la valeur utilitaire dans le discours des répondants

\begin{tabular}{|c|c|c|}
\hline Facette de la valeur & Thème enregistré & Occurrence \\
\hline \multirow{2}{*}{ Efficience économique } & Prix de produit & 48 \\
\cline { 2 - 3 } & Offre promotionnel & 51 \\
\cline { 2 - 3 } & Rapport qualité /prix & 21 \\
\hline \multirow{3}{*}{ Praticité du magasin } & Accessibilité au magasin & 37 \\
\cline { 2 - 3 } & Proximité du magasin & 12 \\
\hline \multirow{2}{*}{ Fiabilité de l’offre } & Gain de temps au sein du magasin & 23 \\
\cline { 2 - 3 } & Diversification de l’offre & 41 \\
\hline \multirow{2}{*}{$\begin{array}{c}\text { Excellence aux bénéfices } \\
\text { utilitaire de l'offre. }\end{array}$} & Disponibilité de produits et services & 5 \\
\cline { 2 - 3 } & Qualité unique perçue par le client du produit & 7 \\
\hline
\end{tabular}

Source : élaboration personnelle. 
Les entretiens auprès des clients des différents magasins marocains ont permis de définir la première composante type de la valeur perçue à savoir la valeur utilitaire. Cette dernière renvoie aux caractéristiques utilitaires de magasin relatives à la fonctionnalité, à la fiabilité et à la qualité perçue. En résumé, nous pouvons dire que, selon les résultats de notre étude, les motifs de fréquentation et de choix d'une grande surface relevant d'une valorisation utilitaire sont :

- Des motifs d'efficience : Liés principalement au prix de produits et services, à la promotion affichée, au bon rapport qualité/prix avec une moyenne de 46 répondants sur 53, soit un pourcentage de $87 \%$. Cela signifie que le consommateur marocain fréquenterait les grandes surfaces essentiellement en raison d’efficience. (Ex. : individu $n^{\circ} 4$ : «je fréquente la grande distribution parce qu'il y a souvent des promotions et ses prix me conviennent ....»).

- Des motifs de fiabilité : Liés à la diversification et la disponibilité des produits, avec une moyenne de 32 répondants sur 53, soit plus de $60 \%$. Donc, le consommateur marocain met en évidence la fiabilité en termes de qualité, de disponibilité et de sélection des marchandises comme raison de son choix de fréquentation de magasin. (Ex. : individu $n^{\circ} 18$ : " je fréquente Marjane parce que sa surface est importante, les allées sont larges et je trouve souvent les produits que je cherche. »).

- Des motifs de praticité : Pour gagner plus de temps et économiser ses efforts, plus de $40 \%$ des répondants soit une moyenne de 24 personnes sur 53, ont choisi de fréquenter les grandes surfaces pour des raisons liées à la proximité et à l'accessibilité des magasins. Le consommateur marocain est donc à la recherche de bonnes affaires tout en minimisant ses déplacements. (Ex. : individu $n^{\circ} 43$ : "je fréquente les grandes surfaces pour faire des achats faciles et gagner du temps. »).

Par contre, les motifs d'excellence ont émergé dans le discours des répondants, avec seulement une moyenne de $23 \%$ des réponses récoltés. Autrement dit, le consommateur marocain préfère consommer des produits et services en se référant à son expérience et/ou au bouche à oreille.

\section{La valeur hédonique}

A partir des entretiens avec les interviewés, nous avons constaté que le mes motifs de fréquentation et de choix des grandes enseignes demeurent liés aussi à son côté hédonique. Nous proposons ci-dessous (voir tableau $\mathrm{n}^{\circ} 4$ ) les deux principales composantes de la valeur hédonique de la typologie d’Holbrook qui sont la valeur esthétique et la valeur ludique : 
Tableau n 4 : L’occurrence de la valeur hédonique dans le discours des répondants

\begin{tabular}{|c|c|c|}
\hline Facette de la valeur & Thème enregistré & Occurrence \\
\hline \multirow{2}{*}{ Valeur ludique } & L'ambiance et le confort du lieu de vente & 27 \\
\cline { 2 - 3 } & La possibilité de tester de nouveaux produits et \\
services & 3 \\
\hline \multirow{2}{*}{ Valeur esthétique } & L'emplacement et la largeur des allées & 33 \\
\cline { 2 - 3 } & Le merchandising & 41 \\
\hline
\end{tabular}

Source : élaboration personnelle.

En nous référant au discours des clients, nous nous apercevons que la valeur hédonique est présente avec un pourcentage de $70 \%$ de la totalité des personnes interviewées. Il apparaît dans leurs réponses que la fréquentation de la grande surface est synonyme de plaisir: se promener, découvertes, opportunité d'apprécier le design et la décoration du magasin, l'atmosphère et l'ambiance suscitée lors des événements comme la période des promotions, la fête de l'Aid, le mois du Ramadan, l'anniversaire de l'enseigne, ...

Nous avons pu donc constater que la fréquentation de l'enseigne pour certains consommateurs est liée beaucoup plus aux attraits visuels et d'ambiance qu'à des raisons d'innovation. (Ex. : individu $n^{\circ} 16$ : il faut que la surface soit importante, les allées larges et l'ambiance du magasin doit être communicative).

\section{La valeur sociale}

Holbrook (2009) définit la valeur sociale comme une catégorie unique de la création et l'amélioration des relations interpersonnelles lors de l'activité de magasinage. A l'instar de cet auteur, nous avons employé le terme lien social que le consommateur cherche à affirmer à travers sa fréquentation du point de vente.

Il est donc nécessaire de distinguer trois types d'interaction sociale (voir tableau $n^{\circ} 5$ ) : les interactions avec les vendeurs, avec les accompagnants et avec les autres personnes présentes dans le magasin.

Tableau $n^{\circ} 5$ : L'occurrence de la valeur sociale dans le discours des répondants

\begin{tabular}{|c|c|c|}
\hline Facette de la valeur & Thème enregistré & Occurrence \\
\hline \multirow{3}{*}{ Lien social } & Interaction avec les vendeurs & 6 \\
\cline { 2 - 3 } & Interaction avec les accompagnants & 9 \\
\cline { 2 - 3 } Statut social & Interaction avec les autres personnes du magasin & 3 \\
\hline $\begin{array}{c}\text { L'image du soi idéal à travers les dimensions } \\
\text { symboliques véhiculées par un produit. }\end{array}$ & 5 \\
\hline
\end{tabular}

Source : élaboration personnelle.

Les facettes de la valeur sociale (faire reconnaissance avec des gens ayant les mêmes centres d'intérêt et accroître son statut unique) acquièrent une faible importance par rapport aux facettes de la valeur utilitaire et 
hédonique. De ce constat, les motifs sociaux (interaction sociale et statut social) ne sont pas distingués comme une raison possible de la fréquentation et du choix du magasin.

Elle apparait avec à peine une moyenne de $11 \%$ chez les consommateurs interrogés. (Ex. : individu $n^{\circ} 7$ : parce que à chaque fois que je visite le magasin, je rencontre par hasard une de mes connaissances, et c'est aussi une occasion de voir mes amis qui travaillent là.), le discours du consommateur marocain renvoie à un capital statutaire fort, qui considère les grandes enseignes comme des lieux qui ne sont pas « accessibles à tout le monde ». Elles sont réservées majoritairement aux personnes d'une catégorie sociale plus élevée.

\section{Les effets de la valeur perçue de magasinage sur la satisfaction des clients}

La satisfaction de la clientèle est l'un des sujets importants desquels se soucient actuellement toutes les entreprises, comme pour le cas des entreprises de la grande distribution. Oliver (1997) définit la satisfaction comme un jugement client, cognitif et affectif, portant sur le service qui lui a été proposé par un fournisseur donné ou encore, selon Bitner et Zeithaml (2000), comme l'évaluation par le client du produit ou du service et sur la capacité de ce dernier à rejoindre ses besoins et ses attentes.

L’analyse des discours des répondants nous a permis de classer les effets de valeur, selon différentes variables influençant la satisfaction des consommateurs. Les résultats sont résumés par le tableau suivant (voir tableau $\left.n^{\circ} 6\right)$ :

Tableau $n^{\circ} 6$ : L'occurrence des facteurs de la valeur perçue en fonction des sources de satisfaction dans le discours des répondants

\begin{tabular}{|c|c|c|}
\hline Facteur & Variable évoqué & Occurrence \\
\hline \multirow{4}{*}{ Facteurs d'ambiance } & Atmosphère & 27 \\
\cline { 2 - 3 } & Implantation des rayons & 23 \\
\cline { 2 - 3 } & Merchandising et design & 41 \\
\cline { 2 - 3 } Economique & Largeur des linéaires & 10 \\
\cline { 2 - 3 } & Rapport qualité, prix et promotion & 35 \\
\hline \multirow{2}{*}{ Service } & Praticité et gain de temps lors de la visite & 23 \\
\cline { 2 - 3 } & Disponibilité et diversité de l'offre & 32 \\
\hline \multirow{3}{*}{ Personnels en contact } & Tester de nouveaux produits & 15 \\
\cline { 2 - 3 } & La confiance envers le magasin & 6 \\
\cline { 2 - 3 } & Lien avec le personnel du magasin & 2 \\
\hline
\end{tabular}

Source : élaboration personnelle.

\section{L'impact des facteurs d'ambiance sur la satisfaction des clients}

Nos résultats montrent une influence des variables de l'ambiance du point de vente sur les états émotionnels des consommateurs marocains 
interviewés. A l’origine de ce résultat, avec une occurrence de 77\%, on trouve le marchandising et design, qui représentent le premier facteur d'influence sur la satisfaction des clients dans les points de vente, en plus de l'atmosphère et l'implantation des rayons qui constituent le deuxième facteur avec un pourcentage de $47 \%$.

Le consommateur marocain se déclare, ainsi, d'autant plus heureux, satisfait et excité face à un merchandising stimulant et un design plaisant. Il n'est pas non plus étonnant que l'atmosphère et l'implantation des rayons conduisent à générer un sentiment de stimulation et de plaisir. En effet, le client marocain a le sentiment d'être heureux et satisfait lorsque la taille du point de vente est jugée agréable, l'emplacement et le déplacement aisés et le rangement du point de vente pratique. (Ex. : individu $n^{\circ} 15$ : oui, je cherche souvent des enseignes belles et modernes dans leurs designs et attrayantes).

\section{L’impact des facteurs économiques sur la satisfaction des clients}

Selon nos résultats, les facteurs économiques sont très présents comme base de la satisfaction dans le discours des répondants. La fréquence des promotions ou l'honnêteté du prix influence fortement la satisfaction et la qualité est un élément clé avec une moyenne de $87 \%$ des personnes interrogées. Sur le plan du rapport qualité, prix et promotion, l'impact sur la satisfaction diffère selon différents attributs notamment l'efficience de l'offre par rapport aux besoins du client est capitale ; la praticité et le gain de temps sont des facteurs clé de satisfaction. Par contre, les pratiques des soldes influent faiblement la satisfaction. C'est un élément secondaire. (Ex. : individu $n^{\circ} 45$ : (...), je ne fais pas toujours confiance aux soldes affichés à l'intérieur du magasin.).

Les résultats de cette étude indiquent également que la perception du client du prix, promotion et qualité de service a des effets complémentaires sur la satisfaction globale du client marocain. Cela a un impact positif sur l'image perçue de l'enseigne. Cela est susceptible de développer une relation durable avec le client. Nous notons par ailleurs que la valeur perçue est corrélée positivement avec la composante de la valeur utilitaire et la stimulation expérientielle.

\section{L'impact des facteurs services sur la satisfaction des clients}

Il ressort de nos résultats que parmi les facteurs influençant positivement la satisfaction du client marocain, on trouve la disponibilité des produits et services et la diversité de l'offre du magasin avec une moyenne de $60 \%$ des personnes interrogées. Cependant, tester les nouveaux produits (innovation) dans les enseignes n'influe pas clairement sur la satisfaction. (Ex. : individu $n^{\circ} 35$ : (...) c'est rare que je teste de nouveaux produits. Je me contente de produits que je connais par cœur.). 
Le consommateur marocain peut être satisfait d'un magasin, en se référant à son expérience ou au bouche à oreille malgré les différentes actions commerciales et marketing mises en place par les grandes enseignes. Notons que dans un environnement concurrentiel, la bonne prestation de services en termes d'accueil et de service après-vente, de réduction des files d'attente devant les caisses et d'entretien d'une relation durable et sociale avec le client sont aussi des sources majeures d'une forte valeur perçue de l'enseigne par le client, qui peut par conséquent influencer le comportement dans le sens positif.

\section{L'impact des facteurs personnels sociaux sur la satisfaction des clients}

D’après les résultats de notre étude, les interactions sociales mises en place au sein des grandes enseignes Marocaines n'affectent pas la valeur perçue et donc le mécontentement des clients. En effet, en matière d'environnement social du magasin, les clients éprouvent un état d'insatisfaction, d'autant plus important que le personnel en contact leur apparaît comme non amical et non serviable. Affirmant que l'attitude des employés et la confiance envers le magasin sont les facteurs les plus déterminants de la satisfaction lors de fréquentation des grandes surfaces. (Ex. : individu $n^{\circ} 11$ : je rencontre des gens de ma connaissance dans le magasin (...), je leur fais confiance surtout pour mes achats importants.).

Par ailleurs, il est évident que la contribution des interactions sociales à la définition d'une forte valeur perçue conduit à une satisfaction de la clientèle. Ce résultat pourrait être à l'origine d'un climat social qui serait donc la personnalité d'une enseigne de distribution qui influence le comportement de son personnel, et par conséquent celui de ses clients. Celuici rend l'expérience de magasinage plus gratifiante et pourrait, par conséquent, participer à la valorisation stratégique du magasin.

\section{Les déterminants de la valeur perçue de magasinage}

L'analyse menée auprès des grandes enseignes de distribution au Maroc et plus précisément à la ville d'Agadir, nous a permis d'identifier les déterminants les plus significatifs de la valeur perçue dans l'acte d'achat des consommateurs, à travers la comparaison des différentes variables sur les valeurs de magasinage. A cet effet, nous voudrions dans ce qui suit (voir tableau $\mathrm{n}^{\circ}$ 7) déterminer les composantes créatrices de valeur, susceptibles de satisfaire et pourquoi pas créer une relation durable avec les clients de l'enseigne. 
Tableau $\mathrm{n}^{\circ} 7$ : L’analyse des déterminants de la valeur perçue de magasinage dans le discours des répondants

\begin{tabular}{|c|c|c|c|c|}
\hline $\begin{array}{l}\text { Type de } \\
\text { Valeur }\end{array}$ & De quoi s’agit-il ? & $\begin{array}{l}\text { Interprét } \\
\text { ation de } \\
\text { rapport }\end{array}$ & Cible & $\begin{array}{l}\text { Occurren } \\
\text { ce }\end{array}$ \\
\hline \multirow{3}{*}{$\begin{array}{l}\text { Valeur } \\
\text { utilitaire }\end{array}$} & $\begin{array}{l}\text { Rapport qualité, prix } \\
\text { et proximité }\end{array}$ & Fort & $\begin{array}{l}\text { Les clients dont l'âge est de } \\
15 \text { à } 44 \text { ans. }\end{array}$ & $37 / 53$ \\
\hline & $\begin{array}{l}\text { Rapport qualité, prix } \\
\text { et choix de magasin }\end{array}$ & Fort & $\begin{array}{c}\text { Les clients dont l’âge est } \\
\text { supérieur à } 30 \text { ans }\end{array}$ & $35 / 53$ \\
\hline & $\begin{array}{l}\text { Rapport qualité, prix } \\
\text { et choix de produit }\end{array}$ & Fort & Les jeunes et les adultes & $41 / 53$ \\
\hline \multirow[b]{2}{*}{$\begin{array}{l}\text { Valeur } \\
\text { hédonique }\end{array}$} & $\begin{array}{l}\text { Rapport qualité, prix } \\
\text { et service }\end{array}$ & Moyen & $\begin{array}{l}\text { Les clients dont l'âge est } \\
\text { supérieur à } 30 \text { ans }\end{array}$ & $23 / 53$ \\
\hline & $\begin{array}{l}\text { Rapport qualité, prix } \\
\text { et nouveaux produits }\end{array}$ & Faible & $\begin{array}{l}\text { Les adultes dont l'âge est de } \\
15 \text { à } 29 \text { ans, surtout en } \\
\text { termes d'origine des } \\
\text { produits }\end{array}$ & $4 / 53$ \\
\hline $\begin{array}{l}\text { Valeur } \\
\text { sociale }\end{array}$ & $\begin{array}{l}\text { Rapport qualité, prix } \\
\text { et interaction social }\end{array}$ & Faible & $\begin{array}{l}\text { Clients plus de } 45 \text { ans qui } \\
\text { valorisent le relationnel }\end{array}$ & $2 / 53$ \\
\hline
\end{tabular}

Source : élaboration personnelle.

Donc, il existe bien une relation entre les caractéristiques du magasin et la valeur perçue des clients : chacune des caractéristiques de l'enseigne a un effet, soit sur la valeur utilitaire, soit sur la valeur hédonique ou bien sur la valeur sociale. Lors des entretiens, de nombreux répondants relient ces dimensions aux différentes variables de la valeur lorsqu'ils font leurs achats au lieu de vente, et se présentent comme suit :

- $\quad$ Plus le rapport est considéré comme « Fort » et jugé satisfaisant, plus le comportement de magasinage a une valeur utilitaire. Traduisant les caractéristiques de la fonctionnalité du magasin (accessibilité et disponibilité des produits), de sa praticité (qualité et rapidité du service) et de son aspect économique (un bon rapport qualité/prix et de gain de temps).

- $\quad$ Le service fourni lors de la fréquentation du magasin et le plaisir qui en résulte sont autant d'éléments qui déterminent la valeur d'achat du client. A cet effet, la composante hédonique de la valeur est observée comme essentielle pour le consommateur marocain.

- $\quad$ Une « Faible » présence des deux variables du rapport, le courage de tester des nouveaux produits et l'interaction sociale: Leur impact sur la valeur perçue serait a priori moins significatifs, et donc, ne sont pas déterminants dans le processus de formation de la valeur perçue du consommateur marocain.

D’après les résultats ci-dessus, nous pouvons dire que ce qui détermine la valeur perçue d'un consommateur marocain, est surtout la combinaison entre plusieurs variables à savoir : Une meilleure qualité à un bon prix, avec la facilité d'accès aux produits ou à l'information. Nous voyons très clairement 
apparaître dans le discours des répondants que plus le rapport, entre les différents déterminants de la valeur perçue de consommateur, est important, plus le comportement de magasinage a une valeur perçue.

\section{Conclusion}

Notre recherche permet de montrer que la valeur perçue est une variable déterminante dans la stratégie des enseignes de la grande distribution. En effet, ce travail portait sur la relation entre les caractéristiques du point de vente et la satisfaction du consommateur, ainsi que sur la mise en évidence de l'effet et des déterminants de la valeur de comportement de magasinage.

Comme nous avons tenté de le démontrer à travers notre recherche, le point de vente n'est plus considéré comme un simple lieu d'achat. Il en ressort différents types de valeurs, telles que les valeurs unitaires, les valeurs hédoniques et les valeurs sociales. Nos résultats sont en mesure de valider cette idée et tendent dès lors à positionner le lieu de vente comme un endroit clé de formulation des stratégies marketing d'une enseigne. Le point de vente doit donc être vu comme créateur de valeur.

Les résultats de ce travail constituent une piste de réflexion importante pour les enseignes de distribution qui sont aujourd'hui face à deux problèmes majeurs : un manque de stratégie de différenciation par rapport à la concurrence et une véritable guerre des prix. Notre étude qualitative s'est déroulée auprès de 53 clients de différentes enseignes de grande distribution de la ville d'Agadir. Le but est de confirmer l'application de la typologie de la valeur d'Holbrook $(1994,1999)$ avec ses trois dimensions dans un contexte marocain.

L'analyse des données qui ont été collectées permette la distinction entre les trois sources de valorisation stratégique : La première est la valeur utilitaire qui renvoie aux fonctions d'usage du magasin. La seconde est la valeur hédonique qui fait que la fréquentation de l'enseigne est synonyme de plaisir dans un climat agréable. La troisième met en évidence le rôle du magasin comme endroit d'interaction sociales et donc de valeur sociales. Précisons également que l'ensemble de cette phase nous a permis d'identifier et de valider les trois composantes de la valeur attachée à l'acte d'achat des consommateurs.

En effet, il est intéressant de constater que les caractéristiques du magasin ont un effet sur la valeur perçue de magasinage. Les résultats de notre recherche empirique, nous ont permis d'identifier ces caractéristiques valorisées par les consommateurs. Au travers de la notion de la valeur perçue, nous avons également abordé des concepts proches tels que la satisfaction du client, et la relation du client avec les différentes enseignes de distribution de la ville d'Agadir. Dans ce sens, les magasins de la grande 
distribution pourront identifier les stratégies créatrices de valeur pour les clients et, ainsi, mettre en place des moyens pour mieux satisfaire et fidéliser leurs clients.

En se référant à cette recherche, la perception de la valeur des enseignes de distribution au Maroc se doit désormais amener les professionnels de métier à tenter de mettre en place plusieurs objectifs comme offrir au client des produits de qualité en rapport avec le bon prix, des services incomparables à ceux de la concurrence, tout en gérant leur magasin dans le respect des aspects de rentabilité, et donner au client une expérience unique en rendant sa visite plus agréable, et ce, en exploitant les résultats des travaux sur la notion de la valeur perçue comme facteur stratégique des enseignes de la grande distribution.

Cependant, différentes limites doivent être signalées. Comme dans toute étude empirique, les résultats obtenus sont en principe des idées (Existantes et/ou nouvelles) et des propositions, qui vont permettre de dégager des pistes de réflexion et d'amélioration. Cette recherche empirique peut être soutenue par l'élaboration d'une échelle de mesure intégrant d'autres facettes de la valeur perçue.

Une autre limite porte sur le choix des grandes surfaces alimentaires. Il serait intéressant de s'appuyer sur d'autres formes de points de vente comme les magasins spécialisés qui peuvent compter sur d'autres formes de valeur. Enfin, si les résultats de cet article s’appliquent aux enseignes de la grande distribution de ville d'Agadir, nous avons signalé que des voies futures de recherche sont envisageables dans le cadre de la valeur perçue, ses sources et ses effets, notamment dans la grande distribution en général.

\section{References :}

1. Amraoui L. (2005), Les effets du prix, de l'image du point de vente et du capital de marque sur la valeur perçue des produits, Thèse de doctorat en Sciences de Gestion, IAE de Toulouse.

2. Akkhlaffou, M., Bouchouar, O., El wazani, Y. \& Souaf Malika (2016). Ethical dimension and performance of public organizations: Effect of the institutionalization of ethical practices on the Moroccan public sector. International Research Journal of Engineering and Technology, IRJET, Volume: 03, Issue: 12.

3. Akhlaffou, M., El wazani, Y., \& Souaf, M. (2016). The Ethical Dimension in the New Public Management: Revisiting the Theory of Accountability, the Case of Public Finances in Morocco. European Scientific Journal, ESJ, 12(31).

4. Aurier P., Evrard Y. et N'Goala G. (2004), Comprendre et mesurer la valeur du point de vue du consommateur, Recherche et Applications en Marketing, 19, 3, 1-20. 
5. Aurier P. et Passebois J. (2002), Comprendre les expériences de consommation pour mieux gérer la relation client, Décisions Marketing, 28, 4, 43- 52.

6. Bali Kalboussi W. (2014), La valorisation du positionnement d'une enseigne de la grande distribution Tunisienne: Une approche exploratoire de la notion de la valeur perçue, Revue Marocaine de Recherche en Management et Marketing n 9-10, p. 341-359.

7. Bakini D. F., Jerbi S., Ben LallounaHafsia H., Effet de la valeur perçue de magasinage sur le comportement du consommateur : Cas d'un point de vente spécialisé, La Revue des Sciences de Gestion 3/2009 (n²37-238), p. 177-185.

8. Bitner, M.J. \& Zeithaml, V.A. (2000). Service marketing: Integrating customer focus across the firm (2nd ed).New York: Irwin McGrawHill Publishing Company.

9. Bonnin G. (1999), L'acte de magasinage : description et interprétation des pratiques spatiales des individus en rayon, Actes $d u$ 15ème Congrès International de L’AFM, Strasbourg, Tome 1, 117136.

10. Bonnin, Gaël (1998), Une typologie des pratiques spatiales des consommateurs en magasin, Actes de la 3ème Journée de Recherche en Marketing de Bourgogne, Dijon, pp.74-88.

11. Bakini Driss F., Ben Lallouna Hafsia H. et Jerbi S. (2009), Effet de la valeur perçue de magasinage sur le comportement du consommateur : cas d'un point de vente spécialisé, La Revue des Sciences de Gestion, 237-238, 177-185.

12. Bouchet P. (2004), L’expérience au cœur de l'analyse des relations magasin-magasineur, Recherche et Applications Marketing, 19, 2,5371.

13. Cottet P., Lichtlé M.C. et Plichon V. (2005), La valeur du comportement de magasinage : effet et antécédents, Actes de l'AFM.

14. Cova B. et Remy E. (2001), Comment et où classer la valeur de lien en marketing ? Actes du 17ème Congrès International de l'Association Française du Marketing, Deauville, AFM, 1-15.

15. Day E. (2002), The role of value in consumer satisfaction, Journal of Consumer Satisfaction, Dissatisfaction and Complaining Behavior, $15,1,22-32$.

16. Filser M. (2000), La valeur du comportement de magasinage. De la conceptualisation aux stratégies de positionnement des enseignes, Actes du 3ème Colloque Etienne Thil, La Rochelle, 1-8.

17. Filser M. (2008), Décision, expérience et valeur de consommation esquisse d'un nouveau cadre théorique pour l'analyse du 
comportement du consommateur, Revue Sciences de Gestion, 64, 2741.

18. Filser, Marc (1994), Le comportement du consommateur, Dalloz, Paris, 426p.

19. Filser M., Plichon V. (2004), La valeur du comportement de magasinage. Statut théorique et apports au positionnement de l'enseigne, Revue Française de Gestion, 1, 148, 29-43.

20. Filser M., Plichon V., et Lambrey B. (2003), La Valorisation de L’Expérience en Magasin : Analyse de L’Adaptabilité d'une Echelle de Mesure de la Valeur Perçue, Cahiers de Recherche GERMATUPRES de VIAE de Tours, 16, 102, 5-24.

21. Hetzel, P. (1996), Décadence et grandeur du marketing de grande consommation : le cas du concept de distribution Nature et Découvertes, in : Annales du management, Association Nationale des IAE, Toulouse, pp.1887-2000.

22. Hetzel, P. (2002), Planète conso. Marketing expérientiel et nouveaux univers de consommation, Editions d'Organisation.

23. Hirschman E.C. et Holbrook M.B. (1982), Hedonic Consumption : Emerging Concept, Methods and Propositions, Journal of Marketing, 46, Summer, 92-101.

24. Holbrook M. B. (1999), Introduction to consumer value, in M.B. Holbrook (coord.), Consumer value : a framework for analysis and research, Londres et New York, Routledge, p.1-28.

25. Holbrook, M. B., (1999), Consumer Value. A Framework for Analysis and Research, Routledge, London, p. 203.

26. Kaabachi S. (2007), La valeur perçue : une variable stratégique pour les enseignes de distribution alimentaire, Acte 2eme Journée du Marketing IRIS, Lyon.

27. Kaabachi S. et Abidi-Barthe A. (2010), Les nouvelles tendances de la grande distribution alimentaire française : une stratégie de création de valeur pour un consommateur postmoderne, 9ème congrès International des tendances marketing, Venise.

28. Kaabachi S. (2007), La valeur perçue : une variable stratégique pour les enseignes de distribution alimentaire, 2ème Journée du Marketing IRIS, La relation client dans les activités de service, Lyon, 15 mars 2007.

29. Kotier P., Keller K., Dubois B. et Manceau D. (2006), Marketing management, 12e édition, Paris, Pearson Education France.

30. Mencarelli R. (2005), Conceptualisation et mesure de la valeur perçue d'un lieu de consommation. Application au domaine du spectacle vivant, Actes du 21ème Congrès International de l'Association Française du Marketing, Nancy, AFM, 1-23. 
31. Merle A., Chandon J.-L. et Roux E. (2008), Comprendre la valeur perçue de la customisation de masse. Une distinction entre la valeur du produit et la valeur de l'expérience de co-design, Recherche et Applications en Marketing, 23, 3, 39-53

32. Oliver, R. L. (1997). Satisfaction: A behavioral perspective on the consumer. New York: Irwin/McGraw-Hill.

33. Philippe A., Yves E. et Gilles N'G. (2004), Comprendre et mesurer la valeur du point de vue du consommateur, Recherche et Applications en Marketing, Vol. 19, No. 3, pp. 1-20.

34. Schmitz G. (2009), The effects of acquisition and transaction shopping value perceptions on retail format usage intentions : an illustration from discount stores, The International Review of Retail Distribution and Consumer Research, 19, 2, 81-101.

35. Siani G., (1998), L’exotisme alimentaire. Un choix de marketing pour les enseignes, La Revue des Sciences de Gestion, Direction et Gestion, n 174.39-51. DOI : 10.1051/larsg : 1998024.

36. Siani G., (2001), Valeurs d'information des attributs marque et enseigne dans l'acte d'achat du consommateur, La Revue des Sciences de Gestion, $\mathrm{n}^{\circ} 187,7-16$.

37. Solomon M., TissierDesbordes E. et Heilbrunn (2005), Comportement du consommateur, Pearson Education France, 6e Edition, p. 586.

38. Souaf M. et Mekkaoui S. (2009), Les motivations de fréquentation des grandes surfaces par les consommateurs marocains: étude exploratoire, La Revue Marocaine de Recherche en Management et Marketing, ${ }^{\circ} 1$, p. 229-244.

39. Rivière A. (2007), La valeur perçue d'une offre en marketing : Vers une classification conceptuelle, CERMAT/ IAE Tours, vol.20/2007.

40. Rivière A. et Mencarelli R. (2012), Vers une clarification théorique de la notion de valeur perçue en marketing, Recherche et Applications en Marketing, vol. 27, n 3, p. 97-123 (revue classée AERES, CNRS, FNEGE).

41. Yves E. et Aurier Ph. (1999), La valeur de l'art. Une approche relationnelle, Actes de la 4ème Journée de Recherche en Marketing de Bourgogne, Dijon, Novembre, pp.4-18.

42. Zeithami V.A. (1988), Consumer perceptions of price, quality, and value: a means-end model and synthesis of evidence. Journal of Marketings. 52. 3. 2-22. 